XXVIII.

\title{
Ueber die Bedeutung des Löwy'schen Phänomens „Blutsteigerung bei Vorbeugen des Kopfes“ für die Diagnose der Arteriosklerosis cerebri.
}

\author{
Von \\ Dr. Julie Bender, \\ Assistenzürztin der Irrenanstalt Frankfurt a. M.
}

Löwy (5) stellte auf Grund von Untersuchungen über den Blutdruck an der Art. temp. eine Steigerung desselben nach 1 Min. langem Vorbeugen des Kopfes als ein spezifisches Symptom der cerebralen Arteriosklerose hin. Als erhöht bezeichnet er einen Druck von $150 \mathrm{~mm} \mathrm{Hg}$ aufwärts. Das Löwy'sche Phänomen, dem eine grosse Bedeutung zuzukommen schien, wurde von anderen Autoren, z. B. Spielmeyer (11) und Kraepelin (3) in die Symptomatologie der cerebralen Arteriosklerose aufgenommen. $\mathrm{Da}$ aber wirkliche Nachuntersuchungen fehlten, so veranlasste Herr Professor Raecke mich, solche anzustellen.

U'm ganz nach den Angaben Löwy's zu verfahren, musste ich mit Bach's Sphygmomanometer arbeiten, obgleich mir wohl bekannt war, dass die damit erhaltenen Werte an Genauigkeit hinter den Zahlen, die neuere Apparate ergeben, zurückstehen. Uebrigens wurden die Druckbestimmungen an der Radialis durch Kontrolluntersuchungen mit Riva-Rocci nachgeprüft.

Ich untersuchte im ganzen 40 Fălle, davon 15 mit verschiedenartigen Psychosen, 25 Fälle von sicherer Arteriosclerosis cerebri, unter letzteren 9 mit positivem Sektionsbefund. Das Löwy'sche Phänomen trat nur in 2 Fällen auf. In allen übrigen Fällen von Arteriosclerosis cerebri liess es sich nicht feststellen.

Nachfolgend gebe ich eine ausführliche Cebersicht uber meine Untersuchungen. 


\section{Gruppe: Hirnerkrankungen, nicht arteriosklerotischer Art.}

Fall 1. Hysterie. Patient J. Sch., 28 Jahre alt, unehelich gehoren, lernte gut in der Schule, in der Jugend gesund, seit 1904 verheiratet. Frau und 3 hinder gesund. 1 Kind mit 9 Monaten an Krämpfen gestorben. Pat., von Beruf Bürstenmacher, verdiente früher $30 \mathrm{M}$. pro Woche, in den letzten Jahren pro Woche nur $20 \mathrm{M}$, hat infolgedessen Nahrungssorgen. Seit 1908 häufig starke Kopfschmerten mit Flimmern vor den Augen, die mehrere Tage anhalten und periodisch auftreten. Häufig Schwindel. Kein Abusus alcoh. Scit Sommer 1912 häufiger Krämpfe mit Bewusstlosigkeit von 5 Min. bis 1/4 Stuncle Dauer, ohne Zungenbiss, ohne Incontinentia urinae et alvi. Inabhängig von den Anfällen grosse Reizharkeit, Alnahme der Denkfähigkeit und des (iediachtnisses.

Siptember 1912 in die Irrenanstalt eingewiesen. In der Anstalt wurden keine hrïmpfo beolachtet.

6. 11. 12. Ta r. 40, nach 1 Minute langem Virlieugen des Kinfes 75.

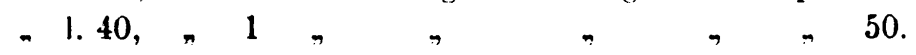

Ra r. $=1.110$.

Bei der lntersuchung Ta r. bcim Vorbeugen des linpfes grosse Angst, kann keine Angahen machen, warum. Sonst keine Beschwerden hei der Lntersuchung.

27. 11. 12. Ta r. 55, nach 1 Minute langem Vurheugen des liopfes j5.

l. $50,-1, \quad, \quad, \quad 60$.

Ra r. $=1.90$.

Sehr müde heim Kopfbeugen, dabei Blutandrang zum liopf - auch ohjektiv - , leichtes unbestimmtes Angstgefühl, ,ich bin jetzt so ganz durcheinander".

Fall 2. Hysterische Haftpsychose. F. K., 35 Jahre alt, in der $J$ ugend gesund, beim Militär gutes Führun@rszeugnis, immer lcicht erreghar, üfters traurig verstimmt, seit 1901 verheiratet, lebte gut mit seiner Frau, war solid und arheitsam. $1906 \mathrm{im}$ Amt als Hilisschaffner, beim Rangieren von einem Waren gegen den Kopf getroffen, fiel hewusstlos zur Erde, geringe äussere Kopfrerletzung, keine hesonderen Folgen, behielt dic stellung. Mär\% 1912 wegen Blutschande, die er an seiner 15 jährigen Tochter verïhte, zu lie. fängnis verurteilt. August 1912 mit einer Haftpsychose in die Irrenanstalt eingrewiesen. Damals verwirrt, ägstlich, die Verwirrtheit geht hald voriber, Pat. zeigt dauernd ein eigenartig läppisches IIesen.

6. 11. 12. Ta r. 70, nach 1 Minute langem Vorbrugen des kopfes (io.

$$
\begin{aligned}
& \rightarrow 1.60,, 1, \quad, \quad, \quad, 60 \\
& \text { Ra r. } \therefore \text { 1. } 90 \text {. }
\end{aligned}
$$

Bei der Untersuchung keine subjektiven Beschwerden.

Fall 3. Epilepsie mit schwachsinn. H. Cl, 25 .lahre alt. Als 3 jähriges lind Scharlarh und Hirnhautentzïndung anschliessend daran rechtsseitige Parese und Neigung zu Erbrerhen. Scit seinem 10. Jahre häufigreres Auftreten ion Anfällen von 1 Minute Dauer mit Bewusstseinsstioruner. Bliisso 
des Gesichts, Augenrollen, Zucken der Finger, keine Aura. Nach dem Infall kein Lnbehagen, keine störung des Bewusstseins. 1901 in die Irrenanstalt eingewiesen, seitdem im . Ibstand ron 1-8 Tagen bis 4 Wochen epileptische Anfälle von 1-10 Vinuten J)auer. In den beiden letzten Jahren seltener Infälle, dabei aher oft mehrtägige postepileptische Zustände mit Ingstgr.fühl, Mattigkeit. leichter Somnolenz. Pat. hat ein stumpies, scheues II esen, wird, wenn man ihn anredet, leicht verlegen, errötet, fängrt an $\mathrm{zu}$ stottern, während seinc Sprache sonst normal ist. Er beschäftigt sich in den letaten Jahren hald mehr hald weniger mit Photographieren, nimmt an Instaltsfesten teil, kleidet sich mit sorgfalt,

12. 8. 12. Mehrere kurz dauernde Infälle, die Petechicn auf der Stirn hinterliessen.

13. 8. 12. Klagt üher Kopfschmerzen rechts, Fingenonmensein des liopfes, schwindel.

Ta r. 55, nach 1 Minute langem Vorbeugen des lopfes 65.

- $1.60,+1,+, \quad, \quad, 65$.

Ra r. $=1.100$.

Lntersuchung verursacht keine besonderen Beschwerden.

Fall 4. Spät-Epilepsie. B. A., $45 \mathrm{Jahr}$ alt. War immer leicht gereizt und ruhelos, schlief wenig, rascher Arbeiter. Seit 1907 Krampfanfälle mit Zungrenbiss, voraufgehender Aura und anschliessendem Zustand von Besinnungrlosigkeit, anfangs im Abstand von 10-4 Wochen, später alle 3 Wochen dazwischen öfters Zustände von Unwohlscin, wobei er blass und blau wurle. Seit Januar 1912 öfters Sehwindelanfiille, begleitet von Verwirrtheitszuständen und undeutlicher Sprache, begeht dabei unsinnige Ilandlungen, verirt sich auf der Strasse; Abnahme des Gedächtnisses. Deshalb Februar 1912 in die Irrenanstalt aufgenommen. Ist hier reizbar, streitsüchtig, hat häufig epileptische. Anfälle, denen tagelang andauernde Verwirrtheitszustände folgen.

25. 7. 12. Ta r. 60, nach 1 Minute langem Vorbeugen des Kopfes 60.

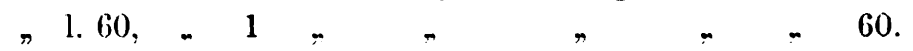

Ra r. $=1.120$.

Am Schlusse der Lntersuchung leichter epileptischer Anfall. Nach dem Anfall Ergebnis nochmaliger Untersuchung an der Ta gleich dem obigen. Untersuchung verursacht keine subjektive Beschwerden.

14. 2.1913 grestorben.

Sektionsbefund: Gefisse an der IIrnbasis zart, frei von Einlagerungen.

Mikroskopischer IIinbefund: Die Mehrzahl der Pial- und Rindengefässe sind zart; nur einzelne der kleineren Rindengefässe zeigen Wucherungsvorgänge von Media und Adventitia, aber nicht stärker als man sie auch sonst bei allen lipileptikern findet. Der gliüse Randfilz ist stark verbreitert und tritt z. T. höckerig über der Oberflache empor. Die Trabantzellen der lianglienzellen sind vielfach vermehrt, auch finden sich hier und da kleine Gliahaufen im Gewebe zerstreut und reihenartiog aufgestellte Gliakerne unkleiden z. T. zartwandige (iefässe. Ilin und wieler trifft man auf sklerotische Ganglien- 
zellen mit korkzieherartigen Fortsätzen. Es handelt sich um eine leichte Arteriosklerose, doch ist das Bild im ganzen mehr das der Epilepsie. Hyaline Fntartung der Gefïsswandungen, Elastikaaufspliterungen, stärkere Schlängelungen, zellarme Zonen finden sich nirgends. Ein arteriosklerotisehe Gehirnatrophie besteht nicht.

Auch bei Elastikafärbung zeigt sich keine stärkere Aufsplitterung und Verbreiterung der Elastika, wie es in anderen arteriosklerotischen Fällen war.

Fall 5. Alkoholismus chronicus. + kopftrauma + Epilepsie. h. W., 42 Jahr alt. Vater des Pat. war Trinker. Pat. in der Jugend gesund. seit 1895 verheiratet. Keine liinder, 1 Frühgeburt. Pat. trank, wenn er Wochenlohn bekam. Schlug im Rausch die Frau, war cifersüchtig, 1898 Frau lebensgrefährlich krank, deshalb schoss sich Pat. eine Kugel in den Kopf, die operativ entfernt wurdc. ${ }_{2}$ Jahr später traten Krämpfe auf mit Bewusstlosigkeit und Schaum vor dem Munde, 1901 wegen der Krämpfe und der Trunksucht in die Irrenanstalt eingewiesen, in der Austalt keine lirämpfe beobachtet, war mehrere Monate hier, trinkt seitdem nicht mehr, hat keine lirämpfe mehr gehabt. Seit seiner 1. Einweisung noch $10 \mathrm{Mal}$ freiwillig auf einige Wochen bis mehrere Monate in der Instalt gewesen, klagte immer über Unlust und Schwächesceühl und Arbeitsunfähigkeit.

27. 11. 12. Ta r. 70 nach 1 Minute langem Vorbeugen des Kopfes 70.

$$
\text { 1. } 70,1, \quad, \quad, \quad, \quad, 60 \text {. }
$$

Ra r. $=1.90$.

Bei der Untersuchung keine subjektiven Beschwerden.

Fall 6. Alkoholismus chron. U.St., 66 Jahre alt. lieine Anamnese 12. 6. 12. Wegen Trunksucht eingewiesen. Ist vollkommen orientiert, klargt über üfters auftretenden Schwindel und Abnahme des Gedïchtnisses.

1. 7. 12. Ta r. 80, nach 1 Ninute langem Vorheugen des hopfes 70.

l. $80, \quad, \quad, \quad, \quad, s()$.

Ra r. $=1.100$.

Bei der Untersuchung keine subjektiven Beschwerden.

Fall $\boldsymbol{i}$. Circuläres Irrescin (scit $40 \mathrm{~J}$.). E. Th., 60.Jahr alt. Wechselnd mit normalen Zeiten, in denen Pat. freundlich, grefälig, ruhig und arbeitsam ist, treten 1) Zustände von grosser Reizbarkeit mit gelegentlicher (Gewalttätigkeit und Personenverkennung auf, daneben 2) Verstimmungszustände, während der sieh Patientin von den Angehörigen und Mitpatienten fernhält, auf Unterhaltungr nicht eingeht. Nic Suizidversuch. Klagt üters über liopfschmerzen und Schwindel.

2. 5. 12. Ta r. 50, nach 1 Minute langem Vorbeugen des Kopfes 55.

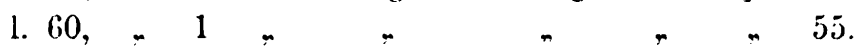

Ra r. $=1.120$.

Bei der Untersuchung ermïdet Pat., sonst keine subjektiven Beschwerden.

Fall 8. Melancholie. 1. B., 62 Jahr alt. Früber gresund, tüchtig, verträglich. In den Wechseljahren üfter Kopfschmerzen, März 1912 aufregende 
Familienangelegenheit. Seitdem ängstliche Verstimmung, mit motorischer Unruhe. Wahnvorstellungen, vorübergehende Erregungszustände. 9. 4.12. Versuch zum Fenster hinaus zu springen, deshalb in die Irrenanstalt eingewiesen. Verhält sich in der Anstalt ruhig, zeigt Neigung zum Weinen, keine Wahnideen, erholt sich nach einigen Wochen, ist ruhig, fleissig, weint nicht mehr, geordnetes Verhalten.

13. 5. 12. Ta r. 120, nach 1 Minute langem Vorbeugen des Kopfes 120.

l. $120, \pi, 1, \rightarrow, \quad, 120$.

Ra r. $=1.105$.

Bei der Lintersuchung keine subjektiven Beschwerden.

2. 6. 12. Ta r. 80 , nach 1 Minute langem Vorbeugen des Kopfes 80 .

l. $80, \quad 1, \quad, \quad, 80$.

Ra r. $=1.100$.

Bei der Untersuchung keine subjektiven Beschwerden.

Fall 9. Dementia praecox. (i. W., 78 Jahre alt. Seit 1864 in der Irrenanstalt. Seit seiner Aufnahme meist stumpfes Verhalten, in den ersten Jahren jedoch gelegentlich streitsüchtig, zeigt öfters Rededrang, schrcibt viel Briefe. Seit 1879 fast unverändert, sitzt immer an gleichem Platz, in gleicher Haltung, spricht fast nichts, grüsst den Arzt immer mit höflicher Verbeugung, hört zuweilen Stimmen.

21. 5. 12. Ta r. 80 , nach 1 Minute langem Vorbeugen des Kinpfes 80.

$$
\begin{aligned}
& \text { l. } 80,-1,10 \\
& \text { Ra r. }=1.160 .
\end{aligned}
$$

Pat. unterzicht sich ruhig der Untersuchung, macht dahei einen vollkommen stumpfen Eindruck, hezüglich suhjektiver Beschwerden kann man keine Angaben erlangen.

Fall 10. Dementia senilis. E. A., 71 Jahre alt. Pat. war immer sehr nervös, immer leicht erregbar und sehr heftig. nie gewalttätig. Betrieb ein Geschäft mit Spielwaren, kaufte sich zu viel Waren ein, hatte keine Ausdauer, überliess der Frau die Führung des Geschäfts, lief ins Wirtshaus, trank wenig, rauchte sehr viel, war einmal ein Jahr lang von der Familie fort. Seit 1910 Schwächegefühl, seit 1911 Schwindelanfälle, seit März 1912 zeitweilig Abnahme des (iedächnisses, Interesselosigkeit, Abnahme des Gewichts. Deshalb in ein allgemeines Krankenhaus eingewiesen, von dort am Ende Mai in die Irrenanstalt verlegt wegen nächtlicher Lnruhe und Desorientiertheit.

In der Irrenanstalt zeigte er leichte Erregbarkeit, motorische Unruhe, Merkfähigkeitsstörung, grosse körperliche Schwäche.

24. 5. 12. Ta r. 80, nach 1 Minute langem Vorbeugen des hopfes 80 .

$$
\text { l. so, }, 1, \quad, \quad, 80 \text {. }
$$

Ra r. - 1. 100.

Bei der lntersuchung keine subjektiven Beschwerden.

31. 7. 12 gestorben.

Sebtionsbefund: (iefässe an der Basis des (iehirns zartwandig, auch in ihren kleineren Verzweigungen. 
Fall 11. Dementia senilis. J. B., 66 Jahre alt. Früher gesund, bis 30. 7. 12 als Schlosser und Schmied tätig, reiste an diesem Tage nach Frankfurt a. M., um seine Kinder zu besuchen, traf nicht bei ihnen ein. Wurde am 8. 9.12 polizeilich in die Irrenanstalt gebracht, da er durch seine Verwirrthcit und Drohungen Passanten gegenüber auf der Strasse auffällig geworden war. Ist örtlich, zeitlich, persönlich desorientiert, bald paraphasische unzusammenhängende Aeusserungen, bald somnolenter Zustand mit lallender Sprache, bald wieder geht er auf Fragen ein und antwortet mit gut artikulierter Sprache.

8. 8. 12. Ta r. 55, nach 1 Minute langem Vorbeugen des Kopfes 55.

1. $70, \pi 1 \pi n \pi 70$.

Ra r. 90, links 120.

Bei der Untersuchung keine subjektiven Beschwerden.

19. 8.12 gestorben.

Sektionsbefund: Atrophia cerebri. Die Gefässwandungen der Hirngefässe sind glatt, keine Einlagerungen.

Fall 12. Dementia senilis. J. A., 73 Jahre alt. Früher Dienstmädchen, seit 11 Jahren wegen Arbeitsunfähigkeit im Versorgungshaus. Bis 18. 7. 12, im übrigen geistig und körperlich gesund. Seitdem nächtliche Unruhe, bann sich nicht besinnen, belästigt die Umgebung durch Fragen und jammert, keine Wahnideen, keine Suizidideen. Am 28. 7. 12 in die Irrenanstalt eingewiesen. Pat. zeigt hier starke Merkfähigkeitsstörung, ist zeitlich desorientiert, hat Bewegungsdrang, ist öfters unrein.

1. 7. 12. Ta r. 70 , nach 1 Minute langem Vorbeugen des Kopfes 70.

l. $70, n 1, \quad, \quad, \quad 70$.

Ra r. $=1.110$.

Bei der Untersuchung keine subjektiven Beschwerden.

Fall 13. Dementia senilis. J. C., 77 Jahre alt. Seit 1907 Abnahme des Gedächtnisses, Merkfähigkeitsstörung, aphasische Störungen, - fand die Worte nicht mehr - . Progressiver Zustand. 1909 lebhafter Wandertrieb, deshalb in die Irrenanstalt gebracht. Jetzt ganz verblödet, zu einer Unterbaltung unfähig, von vorgesprochenen Worten kann sie bis zwei Worte richtig nachsprechen, dann tritt ein Haftenblciben an einer Silbe ein.

19. 7. 12. Ta r. 50, nach 1 Minute langem Vorbeugen des liopfes 55.

l. $55 \pi 1, \quad \rightarrow \quad, \quad \infty 5$.

Ra r. 91, 1. 100.

Bei der Untersuchung keine subjektiven Beschwerden. Pat. ist seit zwei Tagen bettlägerig, ist sehr matt.

Fall 15. Dementia senilis. E. Sch., 77 Jahre alt. September 1912 aus dem Siechenhause wegen nächtlicher Unruhe in die Irrenanstalt verlegt.

Ist vollkommen desorientiert. Gedächtnisschwäche, nächtliche Unruhe; Sprache schwer verständlich, linksseitige Hemiplegie.

23. 9. 12. Ta r. 50, nach 1 Minute langem Vorbeugen des liopfes 50.

1. $55, \rightarrow 1, \times, \quad, 40$.

Ra r. $90,1.70$. 
Bei der Untersuchung Ta r. keine Beschwerden; bei der Untersuchung Ta l. wird das Vorbeugen des Kopfes als anstrengend bezeichnet. Sonst keine Beschwerden.

2. 10. 12 gestorben.

Sektionsbefund: Pachymeningitis chronica, Atrophia cerebri, Encephalomalacia in Capsula interna dextra, Arteriae cerebri zartwandig, keine Einlagerungen.

\section{Gruppe: Fälle von Arteriosklerosis cerebri.}

Fall 16. Manisch depressives Irresein und Arteriosclerosis cerebri. K. Sch., 60 J. alt. April 1870 bis Februar 1871 in der Irrenanstalt in Siegburg. Die hrankengeschichte ergibt: Keine Heredität. Pat. lernte nicht besonders, war später Hausknecht und Schuhmacher. Seit 1869 im Wesen auffallend verschlossen, führt zuweilen irre Reden, mit Zunahme der Verwirrtheit hïrte er auf zu arbeiten, wurde frech und unruhig: kletterte an Baugerïsten herum, lief einem Zug entgegen, gab auf Fragen präzise Antworten. Kam daraufhin in die Irrenanstalt. Sitzt zuweilen zusammengekauert auf der Erde, sagt, der Teufel sei in ihm, ist sonderbar, bald verwirrtes Aussehen, bald träumerisch befangen; wird später durchaus melancholisch geschildert mit Selbstanklagen, Halluzinationen des Geruchs und Gehörs, Beziehungswahn. Diagnose: Melancholie mit Wahn. Februar 1871 als geheilt entlassen.

Februar 1877 bis Juni 1877 in der Irrenanstalt Andernach. Dort wechselndes Verhalten, bald ruhig und zufrieden, bald unstet und gereizt, führt zuweilen hochtrabende Redensarten. Vor der Entlassung ruhig, still, bescheiden, arbeitsam. Als geheilt entlassen.

1912 in Frankfurt a. M. olne Angehörige, keine Anamnese. Wird 5.9.12 wegen starker Cnruhe aus dem Bürgerhospital, wo er eine Pneumonie durchgemacht hatte, in die Irrenanstalt verlert, leidet an chroniseher Nephritis. Verhält sich hier im allgemeinen ruhig, ist zeitlich nicht rollkommen orientiert, klagt über Gedächtnisschwäche, hat wiederholt mehrtägige Zustände von Verwirrtheit. Dic Autoanamnese stimmt im Wesentlichen mit obigen Berichten iiberein.

7. 10. 12. Ta r. 50, nach 1 Minute langem Vorbeugen des liopfes 50.

. l. $50,, 1$, $1, \%$,, 50 .

Ra r. $=1.100$.

Leichte Atembeschwerden beim Vorbeugen des Kopfes, sonst keine Beschwerden bei der Untersuchung.

28. 1.13 grestorben.

Sektionsbefund: Erhebliche Arteriosklerose der (Gefässe an der Hirnbasis. zahlreiche graugelbe, harte Finlagerungen, (iefässe klaffen auf dem Querschnitt.

Mikroskopischer Befund: Sklerosierte Ganglienzellen; Vermehrung der Trahantkerne, starke Schlängelung und Wandverdickung bei den Rindengefässen. Breite, zellarme Randzone. Mässig verdickte Pia ohne Infiltration.

Fall 17. Arteriosclerosis cerebri und Dementia senilis. Th. B.. 79 .I. alt. Am 29. 6. 12 aus dem Versorgungshaus in die Irrenanstalt 
gebracht, war dort unruhig und desorientiert. Hier im allgemeinen orientiert, dement, ofters Schwindel, Schlaflosigkeit, motorische Unruhe. Arteriae radiales et temporales, sehr rigide.

2. 7. 12. Ta r. \$5, nach 1 Minute langem Vorbeugen des hopfes 80.

$, 1.80,, 1, \%, \quad, 80$.

Ra r. $=1.100$.

Das Vorbeugen des Kopfes bei der Lntersuchung wird als anstrengend liezeichnet. 10. 8.12 gestorben.

Sektionsbefund: Atrophia cerebri, chronische Pachymeningitis, starke Arteriosklerose der basilaren Hiruarterien.

Mikroskopischer Befund: Pia hyperplastisch verdickt, nicht infiltriert. Querschnitte der Gefässe stark verdickt. Zellarme Randzone. Ein rerdicktes liefäss der Pia strahlt in die Rinde ein, umgehen von zellarmer \%one. Vermehrte perivaskuläre Gliafasern um die Hirngefässe. Die Kapillaren im Rindenrewebe ohne Infiltration der Wandung. Zu beiden Seiten ron ihnen reihenartigr aufrestellte (iliakerne. Schichtung der (ianglienzellen im allgemeinen erhalten, stellenweise stehen sie ungemein dicht, die Spitzenfortsätze sind nicht immer nach der Peripherie gerichtet.

Fall 18. Dementia arteriosclerotica. F. B., 54 J. alt. 25. 6. 12 wegen Encephalomalacie aus dern Büruerhospital in die Irrenanstalt verlert. Fine Cnterhaltung ist mit dem Pat. nicht mïglich, er macht ganz büde Bemerkungen, oder spricht in einzelnen Silben wie ,ro, no* und lacht dazu blïde, ist meist somnolent, rechtsseitige spastische Hemiplegie.

1. 3. 12. Ta r. 80, nach 1 Minute langem Vorbeugen des hopfes s0.

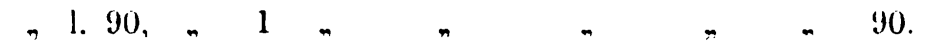

Ra r. $=1.100$.

Sitzen und Vorbeugen des Kopfes wirl vom Pat. als anstrengend bezeichnet. 4. 9.12 gestorben.

Sektionsbefund: Arteriosclerusis graris cerebri. Malacia fere totalis hemisphaerae sinistrae. Leptomeningetis chron. fibrosa.

Mikroskopischer Befund: Blutungen in der Pia, die verdickt, aber nicht infiltriert ist. Die Rindengefïsse haben abnorm breite Wandung, sind seschlängelt und ron zellarmen \%onen umgeben. lianglienzellen chronisch verindert, mit Trabantzellen umgeben. Schnitt erscheint reich an Gilia.

Fall 19. Periodische Melancholie und Arteriosklerose. L. H., 61 Jahre alt. Bis 1887 gesund, damals Zustand ion trauriger Verstimmung. 1900 traurige Verstimmung. Schlaflısigkeit, Suizidwersuch, damals zum ersten $\mathrm{Mal}$ in der Irrenanstalt aufgenommen, ausserdem bis 1908 noch 4 mal mehrere Monate lang in der Irrenanstalt unter ähnlichen Erscheinungen. Seit 1908 dauernd hier. Im Vordergrund steht bei der l'at. der Stimmungrswechsel; sie neigt sehr zu Angstgrefühlen. Hoffnungslosigkeit, Ruhelosigkeit, trauriger Verstimmung ohne (irund, weint leicht, ist für Zuspruch enpfänglich, zeitweise heitere Stimmung, schliesst sich dann gern an andere Patienten an. 
Somatisch: Leichte Verbreiterung des Cor nach rechts, Töne an der Basis akzentuiert, wechselnde Mengen von Eiweiss im Ĺrin.

Frübjahr 1912 häufiges Auftreten von Schwindel, Atemnot, dabei starke Oedeme.

Psychischer Status idem: Hoffnungslosigkeit und Neigung zum Weinen beherrschen das Krankheitsbild.

13. 5. 12. Ta r. 120, nach 1 Minute langem Vorbeugen des hopfes 140.

l. $110, \quad 1, \pi, \pi, 110$.

Ra r. $=1.120$

Bei der Untersuchung keine subjektiven Beschwerden.

31. 7 . gestorben.

Sektionsbefund: Ausgedehnte Arteriosclerosis der Gefässe an der Hirnbasis. Koronarsklerose. Nephritis chron. interstit.

Mikroskopischer Befund des Gehirns: Stark verdickte und geschlängelte Gefässe mit zellarmer Umgebung. An den Gefässen entlang zuweilen Spinnenzellen.

Fall 20. Arteriosclerosis cerebri und Dementia senilis. M. J. i6 Jahre alt. Vollkommen desorientiert, Gedächtnisschwäche, Merkfähigkeitsstörung, kein Schwindel, keine Konfabulationen.

25. 4. Ta r. 100, nach 1 Minute langem Vorbeugen d. Kopfes 100.

l. So, , $1, \%, \quad, 100$.

10. 5. Tar. 120-100, $, 1, \quad, \quad, \quad, 100-110$.

1. $140-120, \% 1 ., \quad, \quad, \quad 80-140$.

Während der Untersuchung wechselnder Befund.

Ra r. $=1.140$.

Untersuchungen verursachen keine instrengung.

7. 7. 12 gestorben.

Sektionsbefund: Gefässe an der Gehirnbasis ziemlich stark erweitert, zartwandig, enthalten stellenweise gelbliche Einlagerungen von kaum merklich erhöhter lionsistenz. Lumen klafft auf dem Querschnitt.

Mikroskopischer Befund: Die von der Pia her an die Rinde einstrahlenden (iefässe stark geschlängelt und verdickt. Bei Toluidinblaufärbung haben selbst die kleinsten liefässe breite, doppelt konturierte Wandungen. Verbreiterte zellarme Randzone: auffallend wenig lierne in der nächsten Umgebung der Gefässe. Pyramidengangrlienzellen auffallend schmal und lang, gleichmässig dunkel gefürbt.

Spitzenfortsätze lang und korkzieherartig greschlängelt, Kern lang und schmal. Vielfach sind (ianglienzellen ron ungewöhnlich zahlreichen Trabantzellen umgeben, auch ihre Fortsätze sind ron solchen eingeschaltet. In der Pia fallen die Quersehnitte stark verdichter Gefässe auf; Infiltration der Pia besteht nicht. die Pia ist hyporplastisch verdickt. Vereinzelte Ganglienzellen zeigen deutliche Pigmentsäcke. 
Fall 21. Arteriosclerosis cerebri und senile Demenz. K. D., 73 Jahre alt. Juli 1912 aus dem Bürgerhospital in die Irrenanstalt eingewiesen wegen Nahrungsverweigerung.

Klagt über Mattigkeit, Atemnot, Mangel an Appetit. Verhält sich im allgemeinen ruhig, isst meist ausreichend, zeitweise erregt.

23. 7. Ta r. 45 nach 1 Minute langem Vorbeugen des Kopfes 45

1. $45,1, \quad, \quad, \quad, 85$

dabei starker Iusten mit Dyspnoe und zyanotisches Aussehen.

Wiederholung 1 Stunde später.

Ta 1. 50 nach 1 Minute langem Vorbeugen des Kopfes 50.

Ra r. $=1.120$.

Der Husten wird als sehr anstrengend bezeichnet, sonst keinc Beschwerden bei der Untersuchung.

20. 8. 12 gestorben.

Sektionsbefund: Feinkörnige, weisse halkeinlagerungen der Gefässe an der Hirnbasis. Lumen klafft auf dem Querschnitt.

Mikroskopischer Befund: Gefässwandung der Rindengefässe stark verdickt, (iliakerne in der Umgebung der Ganglien vermehrt; lange, schwach dunkelgefärbte Ganglienzellen mit langen Ganglienzellfortsätzen und länglichen schmalen Kernen, die sich kaum abheben von dem gleichmässig dunkel gefärbten Protoplasma. Keine Schollen sichtbar.

Fall 22. Arteriosclerosis cerebri und Dementia senilis (bei alter Paranoia). K. F., 89 Jahre alt. Vor 40 Jahren hörte Pat. Stimmen, schirupfte viel mit denselben. Vor 15 Jahren im Wesen auffällig. In den letzten Jahren ist sie ruhiger geworden, zeigte Gedächtnisschwäche, Unruhc nachts. Incontinentia urinae et alvi.

April 1912 wegen allgemeiner Schwäche ins Siechenhaus gebracht. Iuli 1912 von dort aus wegen nächtlicher Erregungszustände in die Irrenanstalt verleırt; ist örtlich, zeitlich, persönlich desorientiert, zeigt Gedächtnisschwäche, Merkfähigkeitsstörung, Mangel an Lrteilsfähigkeit, tagsiiber ruhig, nachts öfters unruhig. Sprache langsam, verwaschen, zeigt Anklang an bulbären Charakter.

Somatisch: Arhythmische Herzaktion, Radialis leicht geschlängelt und verdickt.

27. 7. 12. Ta r. 70, nach 1 Ninute langem Vorbeugen des liopfes 80.

$$
\text { 1. } 60, \quad 1, \quad, \quad, \quad, 70 \text {. }
$$

Ra r. $=1.120$.

Keine subjektiven Beschwerden bei der Lntersuchung.

23. 8. 12. gestorben.

Sektionsbefund: Pachymeningitis chron. Atrophia cerebri. Arterinsclerosis vasor. cerebri baseos. Atheromatosis aortac.

Fall 23. Arteriosclerosis cercbri + Dementia senilis (Phthisis pulmonum). Ki. St., 60 Jahre alt. Scit vielen Jahren nerväs, häufig Kopfschmerzen, seit 1906 oft auffallend im Wesen, starrte of in eine Ecke, gah keine Antwort auf Fragen. 1909 sechs Wochen an einem Lufthurort, fiel bei 
der Rückkehr durch ihr Lachen auf, zeitweise Nahrungsverweigerung, oft Schlaflosigkeit und Angst. 15. 7. 12 mehrstündiger Aufenthalt in der Sonne, danach Zustand ron Verwirtheit, Suixidgedanken. Seitdem $3 \mathrm{mal}$ von Hause wergelaufen, um sich das Leben zu nehmen, $2 \mathrm{mal}$ rom Ehemann wiedergefunden, 27. 7. 12 in die Irrenanstalt grebracht in nassen Kleidern, war in den Main gesprungen. Ist hier anfangs ängstlich, zeigt Bewegungsdrang. Schlaflosigkeit, Gedächtnisschwäche, klagt öfters über Schwindel. Seit septeiiiber verhält sie sich dauernd ruhig, sieht schwach und müde aus, zeigt Neigung zum Weinen, klagt häufig über Parästhesien und Steitigkeiten in den Händen und Unterarmen, I. $>$ r., dabei selten Schmer\%en. Objektiv lässt sich bei let»teren Zuständen livide Verfärbung und Heralisetzung der Hauttemperatur feststellen in der betreffenden Hand.

18. 9. 12. Ta r. 50, nach 1 Minute langem Vorbeugen des liopfes 45.

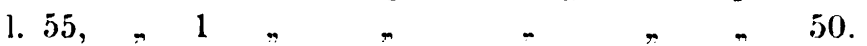

Ra r. $=1.100$.

Beim Vorbeugen des Kopfes klagt Pat. über Atembeschwerden, dabei objektir schnaufende Atmung, keine Dyspnoe.

27. 11. 1․․ gestorben.

sektionsbefund: Leichte atheromatïse Finlagerungen der Gefässe an der Hirnbasis. Phthisis pulmonum.

Mikroskopischer Befund: Im allgemeinen weniger starke Veränderungen als an den übrigen llirnen. Von den kleineren einstrablenden lindengefässen ist die Mehrzahl stark geschlängelt und mässig verdickt. IDie Pia zeigt neben Vermehrungr des Bindegewebes vereinzclte Rundzellenhäufchen.

Um einzelne geschlängelte Rindengefässe der Pyramidenschicht zellarme Zonen, zum Teil ausserordentlich breit. An anderen Stellen (ianglienzellen auffallend dicht und ron massenhaften Trabantzellen umgeben. Die Canglienzellen selbst sind schmal, stark dunkel gefärbt, zuweilen geschlingelte Fortsätze. I)erartige Veränderungen finden sich nur in vereinzelten Zonen und fehlen in einzelnen Schnitten ganz. Rindengefässe nirgends infiltriert.

Fall 24. Arteriosclerosis cercbri + senile Demen\%. M. r. Sch., 65 Jahre alt. Bis April 1910 normal, heiter, selten heftig. Damals verändertes IVesen, sprach auffallend wenig, lief unruhig hin und her, war teilnahmlos. Suizidversueh durch Oeffnen der Gashähne. Seitdem Abasie, Depression. 1. 6. 10 wegen auffallender Apathie in die Irrenanstalt gebracht. Hier zeigt sie nächtliche Unruhe, jammert und stöhnt viel, zerreisst Wäsche, läuft nackt umher, ist unrein.

Somatisch: Dumpfe Herstïne, unsicherer Gang, heine Paraplegie.

Pat. verblïdet nach und nach immer mehr, liegt ruhig zu Bett, keinerlei Klagen.

4. 6. 12. Ta r. 80, nach 1 Minute langem Vorbeugen des Kopfes 80.

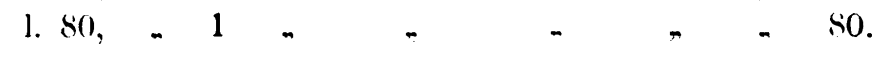

Ra r. $=1.80$. 
Bezüglich subjektiver Beschwerden bei der Untersuchung lässt sich nichts feststellen, da Pat. keine Auskunft gibt. Objektiv keinerlei Anstrengung nachweisbar.

1. 8.12 gestorben.

Sektionsbefund: Ausgedehnte Arteriosklerose der Gefässe an der Hirnbasis. Atrophia cerebri. Hypertrophia cordis, Koronarsklerose, Nephritis interstit. chron.

Mikroskop ischer Hirnbefund: Lichtung vom Fasernetz. Tangentialfasern stark vermindert. Chronische Ganglienzellenveränderung. Schichtung erhalten, stark verschmälert mit geschlängelten Fortsätzen. Struktur nicht mehr recht erkennbar, stark sklerosiert.

Fall 25. Arteriosclerosis cerebri + Alkoholismus. J. K., 65 Jahre alt. Keine Heredität. Normale Entwicklung, von Beruf Küfer, trank immer viel. 1886-1906 Theaterkassierer in Frankfurt a. M. 1906 wegen Arbeitsunfähigkeit entlassen. Weihnachten 1909 Anfall von Atemnot, dabei Angst und Unruhe. Seitdem öfters, besonders nach Alkoholgenuss, Zustände von unbestimmter Angst und Unruhe, läuft dabei planlos in der Stadt umber Pat. besitzt nicht die Kraft, dem Alkohol zu entsagen. Deshalb seit Juli 1911 hier in der Irrenanstalt. Er fühlt sich jetzt im allgemeinen wohl, leidet an Platzangst, verliert angeblich das Gefühl unter den Füssen, wenn er über einen grossen Platz gehen soll. Auch befällt ihn eine enorme Angst, wenn er eine Tür öffnen will und dieselbe klemmt sich.

6. 9. 12. Ta r. 65, nach 1 Minute langem Vorbeugen des Kopfes 60 .

$$
\text { 1. } 85, \quad 1, \quad, \quad, \quad \rightarrow 100 \text {. }
$$

Ra r. $=1.95$.

NB.: Ta l. ist erheblich besser fühlbar als Ta $\mathrm{r}$.

Bei der Untersuchung keine subjektiven Beschwerden.

Fall 26. Arteriosclerosis cerebri + Alkoholismus. E. B., 59 Jahre alt. Vater nervös, mehrere Brüder Trinker. Pat., mittlerer Trinker, kommt Oktober 1912 von selbst in die Irrenanstalt in sehr erschöpftem $\mathrm{Zu}$ stand, obdachlos, klagt über Schwindel beim Bücken und bei Tieflage des Kopfes.

15.10.12. Ta r. 80 plötzl. 100, nach 1 Min. langem Vorbeugen des Kopfes 80 .

$$
\text { 1. } 50 \text {, }
$$

Ra r. $=1.100$.

Bei der Untersuchung der Ta r. gibt Pat. auf Befragen - anlässlich der Drucksteigerung - an, er habe sich beim Aufdrücken der Pelotte erschreckt. Der Gesichtsausdruck bestätigt die subjektive Angabe. Sonst keine Beschwerden bei der Untersuchung.

Fall 27. Arteriosclerosis cerebri + Dementia senilis. M. St., 66 Jahre alt. Immer gesund, früher Wirt, später Hausierer. 27. 8. 12. Schlaganfall, deshalb ins Bürgerhospital gebracht. 9.9.12 wegen grosser Unruhe in die Irrenanstalt verlegt. Zeigt hier leichte Merkfähigkeitsstörung, ist desorien- 
tiert, Schlaflosigkeit. nächtliche Unruhe. Incontinentia urinae et alvi. Rechtsseitige spastische Parese.

17. 9. 12. Ta r. 50, nach 1 Minute langem Vorbeugen des Kopfes 50.

1. $60, \quad 1, \pi, \pi, 60$.

Ra r. $=1.90$.

Bei der Untersuchung keinerlei Beschwerden.

Fall 28. Melancholie + Arteriosclerosis cerebri. L. F., 59 Jahre alt. Väterlicherseits keine Heredität. Geschwister der Mutter: 2 durch Suizid gestorben, ein Bruder wegen Dementia sen. in Anstalt gewesen, ein Bruder Idiot. Mutter gesund; von ihren 3 Kindern: 1 Sohn gesund, 1 Tochter geisteskrank, nicht in einer Anstalt. Pat. früher gesund, 1907 leberleidend, 1910 im Frühjahr leichter Schlaganfall, sprach ganz unverständlich, keine Bewusstlosigkeit, erholte sich rasch, keine Lähmung, Arbeitsfähigkeit ging nach und nach zurück. Gedächtnis nahm ab. November 1911 pensioniert; machte seitdem gelegentlich einige schriftliche Arbeiten, ging im übrigen spazieren, las die Zeitung, Appetit gut, wenig Schlaf, wandert nachts umber. Seit Mai 1912 deprimiert, sprach sehr wenig, Mattigkeit, keine Verwirrtheitszustände, keine Schwindelanfälle. Juni 1912 wegen dieser Beschwerden in die Irrenanstalt aufgenommen.

In der Anstalt zeigt Pat. im allgemeinen nervöse Unruhe, fragt häufig, ob er wieder gesund werde, öfters Angstzustände, selten kurze Verwirrtheitszustände, zuweilen Suizidgedanken. Tagelang macht sich oft eine heitere Stimmung geltend.

3. 7. 12. Ta r. 65, nach 1 Minute langem Vorbeugen des Kopfes 60 .

l. $65, n 1, \pi, \pi, 60$.

Ra r. $=1.100$.

Bei der Untersuchung keine subjektiven Beschwerden.

Fall 29. Dementia arteriosclerotica. A. S., 66 Jahre alt. Am 21. 10. 12 aus einem allgemeinen Krankenhause in die Irrenanstalt überwiesen, weil er an nächtlicher Unruhe litt und seine Saalgenossen mit Fäzes und Urin beschmutzte. Ist vollkommen desorientiert, klagt über öfter auftretenden Schwindel, soll in letzter Zeit öfter auf der Strasse gefallen sein.

Art. temporales et radiales $r$. $=1$. rigide geschlängelt.

25. 10. 12. Ta r. 60, nach 1 Minute langem Vorbeugen des Kopfes 75 ,

$$
\text { l. } 60, \pi 1, \quad, \quad, 60 \text {. }
$$

Ra r. $=1.100$.

Bei der Untersuchung keine subjektiven Beschwerden.

Fall 30. Arteriosclerosis cerebri Alcoholismus chron. A. H., 59 Jahre alt. Zeitlich desorientiert, hat häufiger Schwindel, macht einen erschöpften Eindruck.

28. 9. 12. Ta r. 50, nach 1 Minute langem Vorbeugen des Kopfes 40,

$$
\text { l. } 60,-1, \quad, \quad, \quad, 55 \text {. }
$$

Ra r. $=1.85$.

Bei der Untersuchuug keine subjektiven Beschwerden. 
Fall 31. Arteriosclerosis cerebri + Alcoholismus chron. M. N., 60 Jahre alt. Hat immer stark getrunken, oft sehr jähzornig, immer arbeitsfähig. 1910 Schlaganfall, linksseitige Hemiplegie, seitdem Abnahme des Gedächtnisses. 15.6. 12 zweiter Schlaganfall mit Bewusstlosigkeit und nachfolgendem Zustand von Verwirrung und Sprachstörung. 21. 6. Suizidversuch, deshalb in die Irrenanstalt gebracht. Verhielt sich hier im allgemeinen ruhig, kann sich nur schwer besinnen.

5. 7. 12. Ta r. 65, nach 1 Minute langem Vorbeugen des Kopfes 50.

l. $65, \pi 1, \pi, \pi, 65$.

Ra r. $=1.95$.

Bei der Untersuchung keine subjektiven Beschwerden.

Fall 32. Arteriosclerosis cerebri + Dementia senilis. M. R., $62 \mathrm{Jahr}$ alt. Patientin litt seit Jahren an Schlaflosigkeit, war im Wesen vollkommen normal bis 22. 4. 12. Damals Anfall von Schwäche und Uebelkeit, dem ein 1/2 stündiger Zustand von Bewusstlosigkeit folgte, Bewusstsein blieb 2 Tage getrübt, Sprache dabei undeutlich. Das Bewusstsein kehrte für 1 Tag zurück, dann zunehmende Verwirrtheit mit leichter Benommenheit.

28. 4. 12 in die Irrenanstalt eingewiesen. Benommen, geringe Nahrungsaufnahme; Incontinentia urinae et alvi. Rechtsseitige Parese. Erholt sich allmählich von der Benommenheit. Leichte Sprachstörung, erheblicher Grad von Desorientiertheit, Parese rechts bleiben zurück.

2. 7. 12. Ta r. 70 nach 1 Minute langem Vorbeugen des Kopfes 65

l. $70 \%, \quad, \quad \% 65$

Ra r. $=1.90$.

Untersuchung verursacht keine subjektiven Beschwerden.

Fall 33. Arteriosklerotische Demenz E. H., 65 Jahr alt. 1904 Diabetes mellitus; gleichzeitig damit stellte sich eine depressive Affektlage ein, die immer stärker wurde. 1905 wegen Melancholie mit Suicidgedanken, Verfolgungsideen und Selbstanklagen in die Anstalt aufgenommen. Hier zeitlich und örtlich desorientiert, oft unruhig, litt an Angstzuständen, Verfolgungsideen und Selbstanklagen. Seit 1908 ruhig, nimmt wenig Anteil an der Umgebung, sitzt meist mit verschränkten Armen da, arbeitet nichts. 1912 Status idem. Intelligenzprüfung ergibt erhebliche Demenz.

14. 5. 12. Ta r. 100 nach 1 Minute langem Vorbeugen des Kopfes 100

$$
\text { l. } 100, \quad, \quad, \quad, 100
$$

Ra r. $=1.120$.

Bei der Untersuchung keine subjektiven Beschwerden.

Fall 34. Arteriosklerotische Demenz M. K. 56 Jahr alt. 1904 angeblich nach Blitzschlag Sprachstörung und rechtsseitige Hemiplegie. Nach 6 Wochen Sprache wieder normal. Hemiplegie nicht vollkommen zurückgebildet. lhre Leistungen im Haushalt liessen von da an zu wünschen übrig. 1910 angeblich wieder Schlaganfall mit Sehstörung. 1911 nach dem Tode ibrer Tochter zunehmende Verschlimmerung des Zustandes. Sprache nach und nach ganz 
unverständlich. Pat. leidet seit mehreren Jahren an Kopfschmerzen. Kommit 1912 zur Aufnahme in die Irrenanstalt. Ist sehr dement, zeitweise erregt, Sprache von bulbärem Charakter. Konsonanten werden besonders undeutlich ausgesprochen. Pat. zeigt Neigung zum Weinen, wenn man mit ihr spricht.

Somatisch: Linke untere Extremität wenig paretisch mit leichtem Fussklonus.

21. 11. 12. Ta r. 70 nach 1 Minute langem Vorbeugen des Kopfes 90

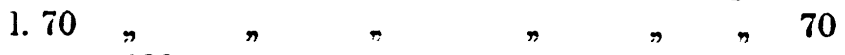

Ra r. $=1.130$.

Untersuchung wird als nicht anstrengend bezeichnet. Pat. weint nach der Untersuchung laut, das Weinen macht einen affektlosen Eindruck.

Fall 35. Depressionszustand, wahrscheinlich Arteriosclerosis cerebri L. K., $61 \mathrm{Jahr}$ alt. Leidet seit Jahren an rheumatischen Beschwerden, besorgt ihren Haushalt immer allein sehr gut. November 1911 fiel Pat. auf der Strasse hin, trug keinen sichtlichen Schaden davon, war nicht bewusstlos. Seitdem leicht ängstlich und aufgeregt, öfters schwindlig. Seit Dezbr. 1911 nicht mehr arbeitsfähig wegen Müdigkeit und Neigung zu Schwindel. 17. Jan. 12. in der Irrenanstalt aufgenommen. Symptome: Angst, Selbstvorwürfe, Schlaflosigkeit, glaubt eine ansteckende Krankheit $\mathrm{zu}$ haben, schliesst sich von den übrigen Patienten ab, ist wortkarg. Urin, Zuckerprobe positiv, 2. 8. 12. Psychisch : Status idem. Urin - Zuckerprobe - negativ.

Ta r. 55 nach 1 Minute langem Vorbeugen des Kopfes 60

$$
\text { 1. } 55
$$

Ra r. $=1.120$.

Bei der Untersuchung keine subjektiven Beschwerden.

Fall 36. Arteriosklerotische Demenz M. M., $60 \mathrm{Jahr}$ alt. Bis zu ihrem 54. Jahre immer gesund. Damals (1906) bei einem akuten Erregungszustand in die Anstalt gebracht. Dabei ideenflüchtig, meist heitere Stimmung, wechselnd mit rasch vorübergehenden Verstimmungszuständen, vielfache Klagen über Kopfschmerzen.

Somatisch: Dumpfe Herztöne, Spur Alb. im Urin. Nach 4 monatligem Aufenthalt in geistig geordnetem Zustand entlassen: war später wiederholt wegen Nierenleiden und Asthma in einem allgemeinen Krankenhaus; Mai 1912 wegen Suizidversuchs - wollte sich die Pulsadern öffnen - wieder in die Irrenanstalt eingeliefert

Symptome: Leicht depressive Affektlage, Gedächtnisschwäche und Klagen über Druck im Kopf.

Somatisch: Cor nach r. und 1. verbreitert, Art. radiales rigide. Chronische Bronchitis, Schrumpfniere. Nach längerem Hiersein stumpfe Affektlage, häufige Klagen über Eingenommensein des Kopfes und Schwindel.

13. 5. 12. Ta r. 130, nach 1 Minute langem Vorbeugen des Kopfes 130.

l. $120, \quad 1, \quad, \quad, \quad, 120$.

Ra r. 120.

1. wegen der Wunde nicht bestimmbar. 
4. 6. 12. Ta r. 75, nach 1 Minute langem Vorbeugen des Kopfes $\mathbf{7 5 .}$

1. $90, \pi 1, \pi, \pi, 90$.

Ra r. $=1.100$.

Bei der Untersuchung keine subjektiven Beschwerden.

Fall 37. Arteriosklerotische Demenz. A. K., 61 Jahre alt. In der Jugend gesund. Mit 51 Jahren (1902) anlässlich eines Wohnungswechsels sehr erregt, ass wenig, hatte die Idee, sie könne nicht essen, könne nicht leben. Dieser Zustand dauerte 2 Monate lang; es trat plötzlich Genesung ein. 1906 im Anschluss an Magenkatarrh Zwangsvorstellungen; glaubte, das Essen sei vergiftet, querulierte und chikanierte viel, kam damals zum ersten $\mathrm{Mal}$ in die Irrenanstalt, war $13 / 4 \mathrm{Jahr}$ dort in unverändertem Zustand, mit vorwiegend ängstlicher Affektlage, Rat- und Hilflosigkeit, zeitweise Erregbarkeit, Misstrauen gegen ihre Umgebung, häufige Nahrungsverweigerung, dabei vollkommen orientiert. In den letzten Wochen ihres Aufenthaltes erhebliche Besserung. August 1912 wieder in die Irrenanstalt eingewiesen. Mässig depressive Stimmung, Sprache langsam, deutlich; nächtliche Unruhe, kein Schwindel, leicht unwillig, muss zum Essen angehalten werden. Incontinentia alvi.

Somatisch: Stark reduzierter Ernährungszustand. Cor ohne Besonderheiten. Venen fast allenthalben stark durchscheinend, zum Theil hervortretend. Art. radiales geschlängelt, rigide.

6. 9. 12. Ta r. 55, nach 1 Minute langem Vorbeugen des Kopfes 50.

l. $50, \pi 1, \pi, \pi, 55$.

Ra r. 80 l. 100.

Vorbeugen des Kopfes wird $r$. $=1$. als anstrengend bezeichnet, sonst keine Beschwerden bei der Untersuchung.

Fall 38. Arteriosklerotische Demenz + Alkoholismus. P. R., 55 Jahre alt. Vater war starker Trinker, Mutter mit 71 Jahren an Schlaganfall gestorben. Pat. als Kaufmann ausgebildet, später 25 Jahre als Hilfsarbeiter am Steueramt, infolge von Trunksucht und Unverträglichkeit nur kleine Stellung, seit 3 Jahren pensioniert. In der Familie unleidlich, brutal gegen Frau und Kinder; 4 Kinder gesund, 5. Kind idiotisch.

Bei der Aufnahme in die Irrenanstalt am 4.6.12 zeitlich und persönlich leidlich orientiert, am nächsten Tag vollkommen desorientiert. Während seines Aufenthaltes in der Anstalt traten wiederholt mehrtägige Verwirrtheitszustände auf, ferner Merkfähigkeitsstörung. Konfabulation; subjektive Klagen bestehen in |Beschwerden über Schwindelanfälle im Bett und ausser dem Bett, häufig Kopfschmerzen, die angeblich im Nacken beginnen und nach oben ziehen $r$. $>1$.

Somatisch: Art. radiales et temporales geschlängelt. Beim Sprechen Vibrieren im Fazialisgebiet; starker Tremor der Zunge. Druckempfindlichkeit der Arm- und Beinmuskulatur, weniger der Nervenstämme. Keine pathologischen Reflexe, Gang breitbeinig, steif, Schwanken beim Augenschluss.

5. 6. Ta r. 75, nach 1 Minute langem Vorbeugen des Kopfes 110.

Ta l. 75, $, 1, \quad, \quad, \quad, 75$.

Rar. $=1.120$. 
Bei der Untersuchung keine subjektiven Beschwerden. Bei einer Unterhaltung im Anschluss an die Untersuchung konfabuliert Pat.

Fall 39. Arteriosclerosis cerebri (nervöse Form). T. E., $59 \mathrm{~J}$. alt. Autoanamnese: Keine Heredität, lernte in der Schule zufriedenstellend, nicht Soldat wegen allgemeiner Körperschwäche. Von Beruf Klempner. Mai 1912 aus der Stellung entlassen, weil er die Feile nicht mehr lange halten konnte, der Arm wurde lahm, bekarn vom Arzt Bäder verordnet, dabei Schwindel. Schwindel besteht schon seit Sommer 1911 beim Bücken, auch oft im Bett beim Umdrehen, Pat. führt das darauf zurück, dass ihm Juli 1911 ein Hebel einer Maschine auf den Kopf gefallen sei. Wegen Schwindels und rheumatischer Beschwerden am 21. Mai 1912 in die Irrenanstalt eingewiesen.

In der Anstalt klagt er über Schwindel, Schmerzen im Fuss und der Wade, auch bald in Schultern und Handgelenk, beschreibt die wechselnd auftretenden Schmerzen als "wie ein eiserner Reif.“ Leichte Ermüdbarkeit, Beine, untere Extremitäten werden steif, in den Fingern oft kein Gefühl. Im Hinterkopf ab und zu Kopfschmerzen.

21. 5. 12. Ta. r. 110, nach 1 Minute langem Vorbeugen des Kopfes 120. 1. $120, \pi 1, \quad, \quad, \quad, 160$. R r. $=1.140$.

Therapie: Bettruhe.

24. 5. 12. Ta. r. 80, nach 1 Minute langem Vorbeugen des Kopfes 80 .

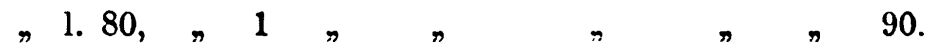

R. $\mathrm{r} .=120$.

Die Untersuchungen verursachen dem Patienten keinerlei Beschwerden.

Fall 40. Hysterie + Arteriosklerose. Th. Z., 63 J. alt. Von Beruf Schneiderin, beobachtete 1872 zuerst nach anstrengender Arbeit abends kurzdauernde Zustände von Aphasie und Bewegungslosigkeit, verbunden mit Sausen im Kopf, wobei Pat. das Bewusstsein nicht verlor. Pat. war immer leicht erregbar und sehr schreckhaft, bei Schreck Neigung zum Aufschreien. Arbeitete mit Erfolg. Sommer 1911 Ohnmachtsanfall, damals Herzleiden konstatiert, einige Wochen später Schlaganfall mit rechtsseitiger Hemiplegie. 1912 wegen Erregungszustände und Suizidideen in die Anstalt eingewiesen.

Pat. zeigt hier Gedächtnisschwäche, Merkfähigkeitsstörung, grosse Reizbarkeit, Neigung zum Weinen, starke Schreckhaftigkeit, stösst dabei laute gellende Tüne aus, ständige Klagen über Mattigkeit, geringe Arbeitsfähigkeit.

Somatisch: Verbreiterung des Herzens, Symptome der Schrumpfniere, Parese des rechten Armes < rechten Beines.

14. 5. 12. Ta. r. 120 , nach 1 Minute langem Vorbeugen des Kopfes 160. ,1.115, $, 1, \pi, 150$. Patient zittert beim Vorbeugen des Kopfes vor Angst, glaubt, dass Ref. ihr dabei heimlich mit dem Instrument etwas tun wolle.

25. 5. 12. Ta. r. 80 , nach 1 Minute langem Vorbeugen des Kopfes 110 . . $100, \pi, \quad, \quad, \quad, 100$. 
Bei der L'ntersuchung der Ta.r. gleiche Angst wie oben, beruhigt sich auf 'Zureden, ist bei der Untersuchung der Ta. l. davon überzeugt, dass ihr nichts geschieht.

4. 6. 12. Ta. r. 60 , nach 1 Minute langem Vorbeugen des Kopfes 70 .

1. $80, \pi 1, \pi, 70$.

Pat. ist bei der Untersuchung vollkommen ruhig, hat keine Angst.

Ra. r. $=1$. schwankt $z$ wischen 120-110.

Bei Fall 40, der anscheinend positives Ergebnis bot, fiel mir bei der Untersuchung auf, dass die Patientin enorm schreckhaft war, sie glaubte, dass ich ihr, wahrend sie den Kopf vorbeugte, mit dem Instrument irgend etwas tun wolle. Sobald es mir gelungen war, sie von dieser Angst zu befreien, blieb das Löwy'sche Phänomen aus. Da ich in der Nervenklinik des städtischen Krankenhauses, wo ich früher långere Zeit arbeitete, hăufig die Beobachtung gemacht hatte, dass Blutdruckbestimmungen nach R. R., die am Tage der Aufnahme des Patienten gemacht werden, sehr hohe Werte angaben, ohne entsprechenden klinischen Befund, wahrend am zweiten oder dritten Tage der Aufnahme, wenn die Patienten sich eingewöhnt hatten, normale und dauernd gleichbleibende Werte auftraten, so nahm ich bei Fall 40 auch an, dass der positive Ausfall im Sinne Lö w y's nicht die Folge der Arteriosclerosis cerebri sei, sondern infolge einer psychischen Komplikation, und zwar Schreck, zustande gekommen sein könnte. Bei Fall 39 trat das Löwy'sche Phänomen auch nur am Tage der Aufnahme auf, während es bei Nachuntersuchungen fehlte.

Meine Vermutung, dass es sich hier um eine psychogene Komplikation handelte, fand eine weitere Unterstützung in den gründlichen Untersuchungen Weber's, die dieser in seinem Werke über den "Einfluss psychischer Vorgänge auf den Körper" niedergelegt hat. Weber (12, S, 66) arbeitete mit dem Plethysmographen und stellte Volumkurven fest. Er sagt, dass eine Steigerung des Blutdruckes einer Zunahme des Volums des dazu gehörigen Körperteils entspricht. Diese Anschauung vertritt auch Herrmann (2) und erklärt dieses durch eine Verengerung der Eingeweidegefässe. Zur Bestimmung der Volumkurven der äusseren Kopfteile machte Weber seine Untersuchungen an der Ohrmuschel. Da die Ohrmuschel ihren Blutzufluss durch Aeste der Art. temporalis und der Art. auricularis post. erhält, beides Aeste der Carotis externa, so ergibt sich, dass die Beobachtungen dort im Prinzip voraussichtlich übereinstimmen dürften mit den Beobachtungen an der Temporalis. 
Es stellte Weber fest (12, S. 108):

Aeussere Kopf- Glieder und ăussere teile Teile des Rumpfes

Bei Entstehung von Bewegunsvorstellung (mit oder ohne Ausführung der Bewegung . . .

Bei geistiger Arbeit . . . .

Bei Lustgefühl $\cdot \cdot \cdot \cdot \cdot \cdot \cdot \cdot \cdot+\quad+$

Bei Unlustgefühl . . . . . .

Bei Spannung . . . . . . . -

$\begin{array}{ll}- & + \\ + & + \\ - & +\end{array}$

+ bedeutet Zunahme, - Abnahme der Blutfülle der betreffenden Körperteile.

Er bemerkt (12, S. 354), dass bei einem Zustand der Erschöpfung oder der Ermüdung der Versuchspersonen beim Eintreten eines psychischen Vorgangs sich die vasomotorische Veränderung in entgegengesetzter Richtung wie im normalen Zustand darstellte. Dies fasst er als ein Hinzukommen einer entgegengesetzt wirkenden Innervation auf. Er nimmt an, dass das Zentrum, das die Impulse, die ihm infolge der Leistung geistiger Arbeit durch die Versuchsperson zufliessen, als konstriktorische Gefässreize weiter gibt, in seinem ermuldeten Zustand nur ein gewisses Mass von diesen Impulsen aufnehmen kann. Die darüber hinausfflessenden Impulse kommen daher dem anderen Zentrum zu gute, das die Impulse, die es erhălt, als dilatatorische Gefässreize weitergibt. Diese pathologische Umkehrung der normalen Volumverănderung beim Eintreten psychischer Vorgånge beobachtete Weber (12, S. 360) besonders bei Basedowkranken, Neurasthenikern und Hysterikern.

Nach diesen Untersuchungen ist es also berechtigt, auch bei unserem Fall 40 die Drucksteigerung mit scheinbar positivem Löwy'schen Phănomen auf die Schreckwirkung zurlickzuführen, und zwar kăme hier die pathologische Umkehrung in Betracht. Wie aus der Anamnese hervorgeht, litt die Patientin seit 40 Jahren an hysterischen Erscheinungen und befand sich bei den Untersuchungen in einem sehr erschöpften Zustande.

Bei Fall 39 wird subjektiv keinerlei Unbehagen bei der Untersuchung angegeben, auch war objektiv nichts dergleichen festzustellen. Auffallend bleibt jedoch, dass das Löwy'sche Phänomen nur am Tage der Aufnahme auftrat, wahrend es spăter fehlte. Man kann in diesem Falle vielleicht annehmen, dass der betreffende Patient bei der ersten Intersuchung mit Aufmerksamkeit der Untersuchung folgte, was eine geistige Arbeit bedeutete, und dass dadurch die Steigerung bedingt wurde. Es kommt auch hier die pathologische Umkehrung in Betracht, 
weil nach der Anamnese der Patient im Juli 1911 von dem Hebel einer Maschine auf den Kopf getroffen worden war und seither bis zu seiner Aufnahme im Mai 1912 uber nervöse Erscheinungen, vor allem leichte Ermüdbarkeit klagte.

Aus diesen Feststellungen ergibt sich also, dass es sich weniger um den Grad der Drucksteigerung als um die Drucksteigerung überhaupt handelt. Ich kann deshalb einige Fälle erwăhnen, bei denen nicht eine Höhe von $150 \mathrm{~mm} \mathrm{Hg}$ erreicht wurde, aber immerhin eine betrăchtliche Steigerung auftrat.

Fall 38 zeigte beim Vorbeugen des Kopfes eine Steigerung von 75 auf 110. Er gab keinerlei subjektive Beschwerden an, wurde aber durch die Untersuchung psychisch sehr beeinflusst und konfabulierte bei einer Unterhaltung, die sich an die Untersuchung anschloss. Es wäre auch hier daher anzunehmen, dass irgend ein psychischer Vorgang durch die Konfabulation bedingt, die Steigerung veranlasst haben könnte. Fall 17 zeigte bei aufrechter Kopfhaltung eine plötzliche Steigerung von 80 auf 100. Auf Befragen gab Patient an, er habe sich beim Aufdrücken der Pelotte erschreckt; der Gesichtsausdruck bestatigte die subjektive Angabe.

Hierzu sei bemerkt, dass nach Weber $(12$, S. 58) bei der Volumkurve der ruhenden, untătigen Versuchsperson, die normalerweise kleine Wellen entsprechend der Herztătigkeit aufweist, gelegentlich Schwankungen vorkommen, die sich durch jähes Abfallen der Volumverminderungen von den anderen unterscheiden. Er sagt: „Es scheint hinreichend sichergestellt, dass diese jähen, plötzlich auftretenden Volumverminderungen mit dem unwillkürlichen Eintreten einer stärkeren psychischen Tătigkeit bei der ruhenden Versuchsperson zusammenhängen, also mit dem plötzlichen Auftreten eines lebhaften Gedankens."

Diese Beobachtung ist hier auf Fall 26 anwendbar, jedoch als pathologische Umkehrung. Auch bei diesem Patienten kommt in Betracht, dass er sich in einem sehr erschöpften Zustand befand. Fall 20 zeigt sowohl bei aufrechter als auch bei gebeugter Kopfhaltung Druckschwankungen von 10 bis $20 \mathrm{~mm} \mathrm{Hg}$, an der Ta. l. eine Schwankung von 80 bis 140. Die Untersuchung verursachte Patientin keine objektiv wahrnehmbare Anstrengung. Es handelt sich hier um eine vollkommen desorientierte Patientin, bei der genauere Feststellungen über ihre psychischen Vorgänge nicht gemacht werden können. Ob hier psychische Vorgänge die Schwankungen verursachten oder dieselben als Undulationen im Sinne Weber's (12) zu bezeichnen sind, bleibt dahingestellt. Bei Fall 21 tritt an der Ta. l. bei vorgebeugtem Kopf eine Steigerung von $30 \mathrm{~mm} \mathrm{Hg}$ auf. Patientin hatte während des Vorbeugens einen Hustenanfall, der starke Dyspnoe und 
zyanotisches Aufsehen verursachte. Eine Nachuntersuchung bei der Patientin in der Ruhe ergab keinerlei Steigerung. Es ist in diesem Falle die Steigerung der Dyspnoe zur Last zu legen, die nach Herrmann (2) pressorische Wirkung hat.

Bei Fall 9 tritt durch das Vorbeugen an der Ta. l. eine Steigerung von $40 \mathrm{~mm} \mathrm{Hg}$ auf. Es handelt sich hier um einen 78 jährigen Patienten, der seit 38 Jahren wegen Dem. praec. in der Irrenanstalt ist und an Mutismus leidet, so dass über seine psychischen Vorgänge also keine Auskunft zu erhalten war. Objektiv bot er wăhrend der ganzen Untersuchung ein vollkommen unverändertes Aussehen. Patient hört zuweilen Stimmen, deren Einfluss eventuell für die Drucksteigerung zur Last zu legen ist. Bei Fall 1, einem Hysteriker, zeigt Ta. r. beim Vorbengen eine Steigerung von 40 auf $75 \mathrm{~mm} \mathrm{Hg}$. Patient gibt an, dass er beim Vorbeugen grosse Angst gehabt habe, kann aber über die Art der Angst keine năheren Angaben machen. Bei einer spăteren Nachuntersuchung, bei der das Vorbeugen Ermüdungserscheinungen veranlasst, verbunden mit einem leichten unbestimmten Angstgefühl, tritt keine Steigerung auf. Erwăhnenswert ist auch Fall 17, bei dem keine Steigernng auftritt, obwohl der Patient das Vorbeugen als anstrengend bezeichnet.

Bezüglich der niederen Druckbefunde von $40 \mathrm{~mm} \mathrm{Hg}$ und höher möchte ich diese nicht dem Apparat zur Last gelegt haben; dass es sich dabei um tatsăchlich wirkliche Befunde handelt, beweist Fall 21, bei dem die pressorische Wirkung der Dyspnoe durchaus in Erscheinung tritt. Es kommen hier vielleicht asthenische Zustände in Betracht, die nach Weber (12, S. 105) besonders bei langandauerndem Kummer auftreten.

Nach Krehl (4) ist die habituelle Blutdrucksteigerung bei Arteriosklerose als eine Reaktionserscheinung des Organismus im Sinne einer Bekämpfung der die Krankheit verursachenden Schădlichkeit aufzufassen. Erfolgreiche therapeutische Massnahmen gegen diese Hypertension bezeichnet er als eine Zerstörung der regulierenden Krăfte des Organismus. Vielleicht handelt es sich bei den asthenischen Zuständen im Sinne Weber's um eine Zerstörung oder Erschöpfung der den Blutdruck bestimmenden Nervenzentren. Alle von mir erwahnten Falle mit dem auffallend niedrigen Blutdruck an der Art. temp. waren hochgradig erschöpfte Patienten.

Hervorzuheben ist, dass die Drucksteigerungen alle bei Patienten auftraten, bei denen neben der Arteriosclerosis cerebri psychische Komplikationen vorhanden waren. Besonders erwähnenswert ist hier auch Fall 1, bei dem keine Arteriosklerose vorliegt. Ein Zusammen- 
gehen der Arteriosclerosis cerebri mit Psychosen ist nichts Auffallendes. Nach Raecke (7) gelten Nervöse, Neurastheniker und Manisch-Depressive wegen ihrer hăufigen Blutdruckschwankungen als vorzugsweise in der Richtung gefăhrdet, dass bei ihnen später eine arterioskleriotische Hirnerkrankung hinzutreten kann.

Betrachten wir nunmehr unter dem gleichen Gesichtspunkte die Falle Löwy's mit positivem Blutdrucksymptom, so finden wir in der Anamnese bei allen seinen Fällen psychische Komplikationen, z. B. melancholisch-hypochondrische Depression, Hysterie, konstitutionelle Neurasthenie und bei einem Fall Diabetes, der bekanntlich haufig mit nervösen und psychischen Störungen einhergeht.

$\mathrm{Zu}$ beachten bleibt noch, dass die Steigerungen sowohl bei Löwy's als auch bei unseren Untersuchungen fast ausschliesslich bei vorgebeugtem Kopfe auftraten. Diese Erscheinung liesse sich wohl darauf zurückführen, dass bei vorgebeugtem Kopfe psychische Vorgänge lebhafter werden, da die unwillkürliche Ablenkung, die durch freies Umherblicken entsteht, wegfallt.

Es erscheint nicht ausgeschlossen, dass durch die psychische Erregbarkeit eines Patienten das Ergebnis beeinflusst wird. Jedenfalls beweist unsere Nachpriffung, dass die Blutdrucksteigerung in der Temporalis bei Vorbeugen des Kopfes nicht ohne Weiteres einer arteriosklerotischen Erkrankung der Kopfgefăsse und der -des Gehirns zur Last gelegt werden darf.

So interessant also auch die Untersuchungen Löwy's sind, so eignen sie sich doch in keiner Weise zur Differentialdiagnose des Frühstadiums der Arteriosclerosis cerebri gegenüber der Neurasthenie und Depression bei manisch-depressivem Irresein. Der positive Ausfall beruht meines Erachtens nicht auf Arteriosclerosis cerebri, sondern kommt in erster Linie durch eine psychische Komplikation zustande und findet sich daher mindestens ebenso oft bei rein funktionellen Störungen.

\section{Literaturverzeichnis.}

1. Breiger, Plethysmographische Untersuchungen an Nervenkranken. Zeitschr. f. d. ges. Neurol. und Psych. Bd. 7. S. 413. Original.

2. Hermann, Physiologie. S. 520. 13. Aufl. 1905.

3. Kraepelin, Psychiatrie. 2. Bd. S. 589. 8. Aufl. 1910.

4. Krehl, Ueber die krankhafte Erhöhung des arteriellen Druckes. Deutsche med. Wochenschr. S. 1572. 1905.

5. Löwy, Ein Blutdrucksymptom der zerebralen Arteriosklerose. Prager med. Wochenschr. 30. Jahrg. Nr. 2-3. 1905. 
1152 Dr. Julie Bender, Blutdracksteigerung bei Vorbeugen des Kopfes.

6. Müller, O., Ueber Arteriosklerose. Deutsche Klinik. 12. Bd. S. 329.

7. Raecke, Die Frühsymptome der arteriosklerotischen Gehirnerkrankung. Referat. 37. Versammlung Südwestdeutscher Neurologen und Irrenärzte am 8. Juni 1912 in Baden-Baden.

8. Resnikow u. Dawidenkow, Beiträge zur Plethysmographie des menschlichen Gehirns. Zeitschr. f. d. ges. Neurol. und Psych. 4. Bd. S. 129. Original.

9. Rudolf, Ueber den erhöhten Blutdruck bei Arteriosklerose. Münchener med. Wochenschr. S. 588. 1911.

10. Sawada, Blutdruckmessungen bei Arteriosklerose. Deutsche med. Wochenschr. S. 425.1904.

11. Spielmeyer, Handbuch der Psychiatrie. Spez. Teil. 5. Abt. S. 126.

12. Weber, Einfluss psychischer Vorgänge auf den Körper. 1910. 